\title{
Correction to: Preliminary Results from the FOGSI's National Registry on Pregnancy with COVID-19
}

\author{
Alpesh Mahendrabhai Gandhi ${ }^{1}$. Atul Morarji Ganatra ${ }^{2,3} \cdot$ Parikshit Tank $^{4}$
}

Received: 16 September 2021 / Accepted: 16 September 2021

(c) Federation of Obstetric \& Gynecological Societies of India 2021

\section{Correction to: \\ The Journal of Obstetrics and Gynecology of India (July- August 2021) 71(4):361-368 https://doi.org/10.1007/s13224-021-01537-3}

The office bearer names and Managing Committee have been missed in the acknowledgement section of the original publication of the article.

The complete acknowledgment section should read as below:

Acknowledgements We would like to acknowledge FOGSI and each hospital department participating in the FOGSI COVID registry. Specifcally, we would like to acknowledge following FOGSI members: Dr. Reena Jatin Wani (reena.wani@redifmail.com), Dr. Shrinivas Gadappa (gadappashrinivas@gmail.com),Dr. Samir Ramesh Pradhan (drsamirpradhan@gmail.com), Dr. Nozer Khursed Sheriar (nsheriar@gmail.com), Dr. Ajith S (drajiths@hotmail. com), Dr. Parul Tushar Shah (parulshah11@ @otmail.com), Dr. Ajesh N Desai (andesai38@gmail. com), Dr. Ashish Vishnu Gokhale (dravgokhale@yahoo.com), Dr. Saroj Singh (drsarojsingh@ @otmail.com), Dr. Rajesh Yashwant Mhatre (rmhatre120169@gmail.com), Dr. Raina Chawla (raina. chawla@gmail.com), Dr. Vineeta Gupta (vineetahims@

The original article can be found online at https://doi.org/10.1007/ s13224-021-01537-3.

Atul Morarji Ganatra

ganatra.atul@gmail.com

1 Arihant Women's Hospital, Ambica Anandmayi Society, Near Over Bridge, Chandlodiya, Near Manikaka Chawk, Chandlodiya, Ahmedabad, Gujarat 382481, India

2 Dr.R.J.Ganatra Nursing Home, Omkar Lal Building, Opposite Mulund West Railway Station, Mulund (West), Mumbai, Maharashtra 400080, India

3 Fortis Hospital, Mulund (West), Mumbai, Maharashtra 400080, India

4 Ashwini Maternity and Surgical Hospital, Mahatma Gandhi Road, Ghatkopar (East), Mumbai, Maharashtra 400086, India yahoo.co.in), Dr. Vandana Kanumury (kanumuryvandana@ gmail.com), Dr. Yamini Nilesh Trivedi (yamilesh2@ yahoo. com), Dr.Ashvin Dayalal Vachhani (avachhani74@gmail. com), Dr. Jitesh Shah (avachhani74@ gmail.com), Dr. Uma Pandey (uma.pandey2006@yahoo.com), Dr. Anita Kant (dranitakant@yahoo.com), Dr.Kasturi V Donimath (dkasturiv@ redifmail.com), Dr. Poonam Verma (poonam@ mgims.ac.in), Dr. Anita Abhijeet Soni (anita.soni8@ hotmail. com), Dr. Prabhadevi Kodey (drprabhakodey@gmail.com), Dr. Nidhi Gupta (drnidhigupta19@gmail.com), Dr. Sarita Agrawal (drsaritaagrawal@yahoo.com), Dr. Nalini Inderpal Anand (docnalini.anand@ gmail.com), Dr. Vrunda Joshi (vrunda2007@ redifmail.com), Dr. Tripti Nagaria (triptinagaria@gmail.com), Dr. Kavita N. Singh (drkavitasingh67@ gmail.com), Dr. Manpreet Kaur Tehalia (dr_mjtehalia@ yahoo.co.in), Dr. Datta Manohar Panandikar (dattamp63@ gmail.com), Dr. Bharti Maheshwari (bhartinalok123@ gmail.com).

The authors and the FOGSI National Registry Working Group would like to acknowledge the facilitatory role of the FOGSI Office Bearers and the FOGSI Managing Committee for the year 2020 to 2021. Specifically, we would like to acknowledge the following individuals with their designations for 2020 to 2021 Dr. Jaydeep Tank (Secretary General), Dr. Anita Singh, Dr. Archana Baser, Dr. Ragini Agrawal, Dr. T. Ramani Devi (Vice President), Dr. Madhuri Patel (Deputy Secretary General), Dr. Suvarna Khadilkar (Treasurer), Dr. Sunil Shah (Joint Secretary), Dr. Nandita Palshetkar (Immediate Past President) and Dr. S. Shantha Kumari (Incoming President Elect).

Publisher's Note Springer Nature remains neutral with regard to jurisdictional claims in published maps and institutional affiliations. 